\title{
Optimization and design of the vulcanization press sandwich pressure plate parameters
}

\author{
Milan Vaško ${ }^{1, *}$, Marián Handrik ${ }^{1}$, Peter Kopas $^{1}$, Bohuš Leitner ${ }^{2}$ \\ ${ }^{1}$ University of Žilina, Faculty of Mechanical Engineering, Department of Applied Mechanics, \\ Univerzitná 8215/1, Žilina, Slovak Republic \\ ${ }^{2}$ University of Žilina, Faculty of Security Engineering, Department of Technical Sciences and \\ Informatics, 1. mája 32, Žilina, Slovak Republic
}

\begin{abstract}
The goal of this paper is to present the optimization of the vulcanization compactor pressure plate construction design. The basic factor for the mechanism functionality is the required stiffness compliance. The total construction stiffness has an influence on the vulcanization form tightness. Several solutions of the pressure plate have been proposed in the initial phase. Each proposal has been optimized in order to minimize the pressure plate weight meeting the conditions of the maximum displacement and the maximum allowed stress. The best solution has been chosen on the base of the evaluation afterwards. The multisoftware approach using ADINA and MATLAB software was used to perform FE analyzes.
\end{abstract}

Keywords: ADINA, MATLAB, optimization algorithms, Nelder-Mead optimizing algorithm, sandwich pressure plate, FE analysis

\section{Introduction}

Structures exposed by extreme load are often characterized by a large weight of the carrier parts. This is currently removed by various design modifications. These adjustments require consistent control in terms of strength and fatigue life. By using FEM, it is possible to obtain deformations and stresses distribution in the structure and identify the critical locations. The results of the analysis are closer to reality, assuming a suitable computing finite element mesh $[1,2]$.

Contradictory requirements are placed on the pressure plates in the production of compactor parts. The plates must satisfy strength and deformation conditions in relation to the operational requirements. These often lead to the design of massive high weight plates. This deficiency can be eliminated by designing a sandwich plate that retains the same stiffness and deformation properties and meets the strength requirements. This approach will be applied to a specific construction in the article $[3,4,5]$.

\footnotetext{
* Corresponding author: milan.vasko@fstroj.uniza.sk Reviewers: Tomasz Domański, Michal Šofer
} 


\section{Formulation of the optimization problem}

The aim of the contribution will be based on the optimization process to design a suitable thickness of the upper and lower plates of sandwich construction $X_{1}$, the thickness of the ribs $X_{2}$ and the distance between plates (rib height) $X_{3}$ (Fig. 1) $[4,6]$.

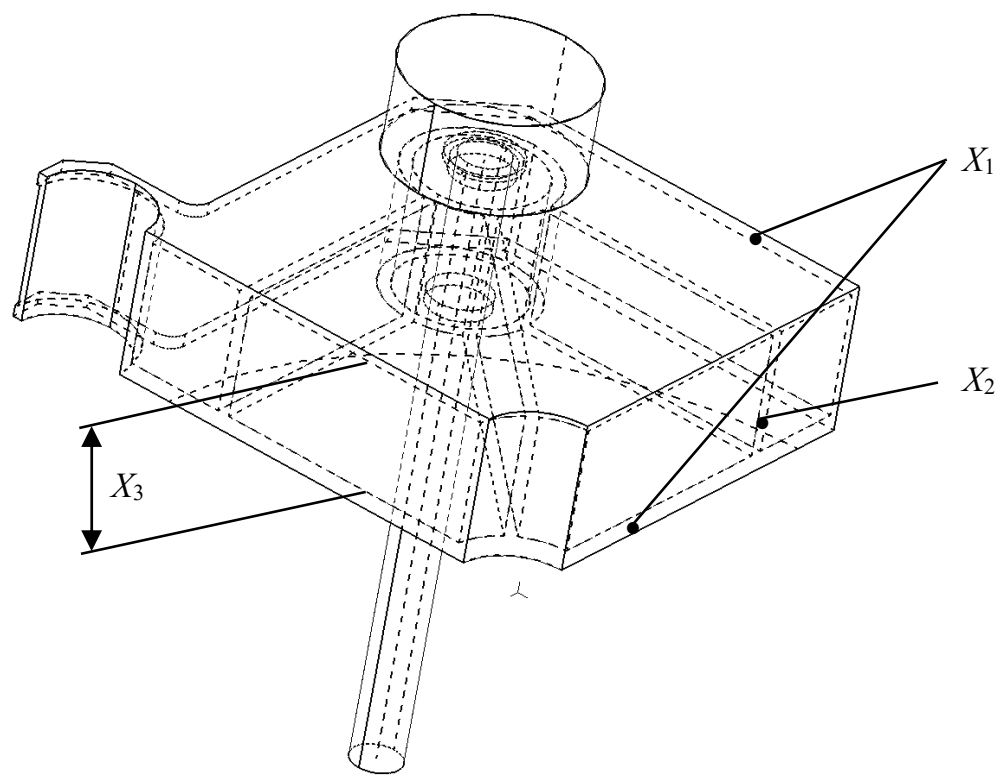

Fig. 1. Model of sandwich pressure plate with optimization parameters

Applying the above-mentioned requirements for the weight, stiffness (deformation) and strength (stress), the optimization problem was formulated as follows

$$
F\left(X_{1}, X_{2}, X_{3}\right)=m=\rho \cdot \sum_{i=1}^{n} V_{i}\left(X_{1}, X_{2}, X_{3}\right) \rightarrow \min ,
$$

where $m$ is the weight of the structure determined by FEM analysis from the volume of the model and the density of the material under the following conditions:

- the deformation and stiffness condition:

$$
U_{\max }\left(X_{1}, X_{2}, X_{3}\right)-U_{D O V} \leq 0,
$$

- the strength condition:

$$
\sigma_{\max }\left(X_{1}, X_{2}, X_{3}\right)-\sigma_{D O V} \leq 0,
$$

- the geometrical condition:

$$
\begin{aligned}
& 0.01 \leq X_{1} \leq 0.2 \\
& 0.01 \leq X_{2} \leq 0.2 \\
& 0.10 \leq X_{3} \leq 0.4
\end{aligned}
$$

The $U_{\max }, U_{D O V}$ parameters represent the maximum calculated and allowed deflection. The $\sigma_{\max }, \sigma_{D O V}$ parameters represent the maximum calculated equivalent stress, respectively its permissible value. 
The new objective function has been built with respect to restrictive conditions on the principle of the method of penalty functions in the form

$$
F\left(X_{1}, X_{2}, X_{3}\right)=\left\{\begin{array}{c}
\rho \cdot \sum_{i=1}^{n} V_{i} \rightarrow \min (\text { if conditions } 2,3 \text { and } 4 \text { are valid }) \\
\lambda(\text { in other case }),
\end{array}\right.
$$

where the penalizing coefficient is $\lambda=2000$ if one of the constraints is not met, otherwise $\lambda=0$. The objective function (5) was optimized by an improved simplex NelderMead algorithm programmed in MATLAB [7, 8].

\section{Modified simplex optimization method}

The original sequential simplex method was designed by Spendley, Hext and Himsworth [9] and then enhanced by Nelder and Mead [10]. The original proposal of the method consisted of applying a regular geometrical shape called simplex with $n+1$ vertices in $n$-dimensional space $\mathrm{R}^{n}$, as shown Fig. 2.

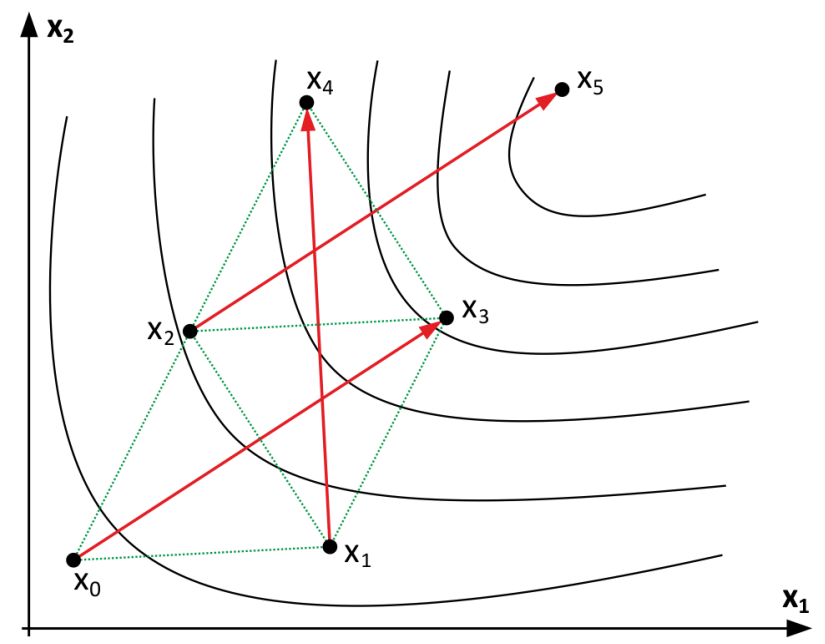

Fig. 2. Graphical representation of the method's algorithm

The improvements suggested by Nelder and Mead also begin with a regular simplex that, however, changes during the computation according to the convenience of the direction in which the search process is executed.

The simplex vertices can be defined by the beginning and the points along each of the $n$ coordinate directions. Such simplex cannot be geometrically symmetrical. The following equations are suggested in reference [9]. To calculate the position of vertices of a uniform simplex of the size and in $n$-dimensional space, we use the following relations

$$
\mathbf{x}_{j}=\mathbf{x}_{0}+p \cdot \mathbf{e}_{j}+\sum_{\substack{k=1 \\ k \neq j}}^{n} q \cdot \mathbf{e}_{k}, \quad j=1, \ldots, n
$$

with

$$
p=\frac{a}{n \sqrt{2}}(\sqrt{n+1}+n-1) ; \quad q=\frac{a}{n \sqrt{2}}(\sqrt{n+1}-1)
$$


where $\mathbf{e}_{k}$ is the unit vector along the $k^{\text {th }}$ coordinate direction, and $\mathbf{x}_{0}$ is the initial reference point. For example, for a problem in the assumed two-dimensional space, equations (6) and (7) determine an equilateral triangle with sides $a$. When the simplex is defined, the function $F$ is evaluated in each of the $n+1$ vertices $\mathbf{x}_{0}, \mathbf{x}_{1}, \mathrm{~K}, \mathbf{x}_{n}$. Let $\mathbf{x}_{h}$ and $\mathbf{x}_{l}$ denote the vertices where the function $F$ acquires its maximum and minimum in this sequence, and $\mathbf{x}_{s}$ denote the place where the vertex acquires the second highest value. The simplex method deletes the vertex $\mathbf{x}_{h}$ and replaces it with the point where the function $F$ has the lowest value. This is achieved in three operations called reflection, contraction and expansion. Operation reflection generates a new point $\mathbf{x}_{r}$ along the line of the passing from $\mathbf{x}_{h}$ to the centre of gravity $\overline{\mathbf{x}}$ of the remaining points, which is defined as follows:

$$
\overline{\mathbf{x}}=\frac{1}{n} \sum_{i=0}^{n} \mathbf{x}_{i}, \quad i \neq h .
$$

The vertex point at the end of reflection is calculated as

$$
\mathbf{x}_{r}=\overline{\mathbf{x}}+\alpha \cdot\left(\overline{\mathbf{x}}-\mathbf{x}_{h}\right)
$$

where $\alpha$ is a positive constant called reflection coefficient that is usually considered unitary. Each positive value of the reflection coefficient in equation (9) ensures that $\mathbf{x}_{r}$ is situated on the opposite side of $\overline{\mathbf{x}}$ than $\mathbf{x}_{h}$. If the function value in this new point $F_{r}=F\left(\mathbf{x}_{r}\right)$ meets the condition $F_{l}<F_{r}<F_{s}$, then $\mathbf{x}_{h}$ is replaced by the value $\mathbf{x}_{r}$ and the process is repeated with this new simplex.

Conversely, if the value of the function $F_{r}$ at the end of the reflection is lower than the lowest value of the function $F_{l}=F\left(\mathbf{x}_{l}\right)$, then there is a possibility that we can still reduce the function by moving further along the same direction. We are looking for a more accurate point $\mathbf{x}_{e}$ with the expansion method using the relationship

$$
\mathbf{x}_{e}=\overline{\mathbf{x}}+\beta \cdot\left(\mathbf{x}_{r}-\overline{\mathbf{x}}\right),
$$

where $\beta$ is the expansion coefficient that often takes the value of 2 (or 1.95). If the value of the function $F_{e}$ is smaller than the value at the end of the reflection step, then replace $\mathbf{x}_{h}$ with the value $\mathbf{x}_{e}$ and repeat the process with a new simplex. Otherwise, if the reflection leads to the function value equal or greater than $F_{r}$, then create a new simplex by replacing $\mathbf{x}_{h}$ with the value $\mathbf{x}_{r}$ and continue the process further.

If the reflection process leads to the point $\mathbf{x}_{r}$ so that $F_{r}<F_{h}$ is true, then replace $\mathbf{x}_{h}$ with the value $\mathbf{x}_{r}$ and perform the contraction operation. Conversely, if $F_{r} \geq F_{h}$, then perform contraction without any replacement using the relation

$$
\mathbf{x}_{c}=\overline{\mathbf{x}}+\gamma \cdot\left(\mathbf{x}_{h}-\overline{\mathbf{x}}\right)
$$

where $\gamma$ is the contraction coefficient $(0<\gamma<1)$. The coefficient usually takes the value of 0.5. If $F_{c}=F\left(\mathbf{x}_{c}\right)$ is greater than $F_{h}$, all points are replaced with a new group of points

$$
\mathbf{x}_{i}=\mathbf{x}_{i}+\frac{1}{2}\left(\mathbf{x}_{l}-\mathbf{x}_{i}\right) ; \quad i=0,1, \ldots, n
$$

and the process starts from the beginning with the new simplex. Or simply replace $\mathbf{x}_{h}$ with $\mathbf{x}_{c}$ and the process with this simplex will start from the beginning.

The operation in (12) causes that the distance between the old simplex points and the point with the lowest value of the function is reduced by half, and therefore it is sometimes 
referred to as the contraction operation. As a convergence criterion to terminate the algorithm, Nelder and Mead [10] suggested the following equation

$$
\left\{\frac{1}{1+n} \cdot \sum_{i=0}^{n}\left(F_{i}-\bar{F}\right)^{2}\right\}^{1 / 2}<e
$$

where $e$ is the pre-defined condition of convergence, and $\bar{F}=F(\overline{\mathbf{x}})$.

\section{The computational model for analysis}

The entire computational model can be in terms of algorithmization divided into two tasks:

- compilation of the parametric FEM model for the ADINA calculation software and related files for evaluation of the results [11],

- creating an objective function in MATLAB [4, 12].

The high time requirement is assumed based on the character of the optimization task, and therefore it is necessary to create models with minimal computational demands $[13,14]$. Therefore, when creating a FEM model, the double symmetry of construction and a few simplifications were used. Simplifications consist in the rigid connection of the pressure plate with the hydraulic cylinder, which effects the closing force $F=2 \mathrm{MN}$. This design node would require a contact solution in case of requiring an exact solutions task [15].

Another simplification used is the assumption of the uniform pressure load at the point of contact of the pressure plate with the mold container. The elongated piston rod has taken away degrees of freedom at its free end in the direction of its axis. In the analysis, a linear material model with the following properties was used:

- Young's modulus of elasticity

- Poisson's number

- density of the material

$$
\begin{gathered}
E=2.1 \cdot 10^{11} \mathrm{~Pa}, \\
\mu=0.3, \\
\rho=7850 \mathrm{~kg} / \mathrm{m}^{3} .
\end{gathered}
$$

Quadratic tetrahedron type elements were used in the FEM model. The total number of equations exceeded 350000 . An efficient iterative solver was used to solve this system of linear equations. Duration of one FEM analysis lasted maximum of 3 minutes (including generating the model). The efficiently assembled model has made it possible to perform an optimization task from a single start point in the short time, as well as mapping the whole area of acceptable solutions to identify local extremes of the objective function [16].

The target function algorithm consists of the following steps:

- evaluation of the restrictive conditions of the equation (4) - in the event of their failure penalizing the objective function and the completion of the evaluation of the objective function [17],

- if the conditions of equation (4) are met, then the input file for ADINA is generated with current values of the optimization variables [11],

- generating the FEM model and launching the task solution,

- evaluation of results - determination of weight, maximum equivalent stresses according to von Mises theory, maximum plate deflection and evaluation of fulfillment of the conditions according to relations (2) and (3) $[18,19]$,

- in the event of non-fulfillment, the target function is penalized, otherwise the target function value is determined based on the calculated weight of the structure.

The shape of the target function according to equation (5) and its evaluation procedure is suggested for the following reason - if the geometric constraints for the area of permissible solutions (4) are not met, the FEM analysis is not required. 


\section{Results of the optimization process}

The optimization process had to be carried out in two steps due to the large number of local extremes in the investigated area. Therefore, the authors decided to map out a set of optimal solutions and find the best starting step within the permissible interval. Figures 3 and 4 show the map of values for the optimization variables at the start point and at optimum.

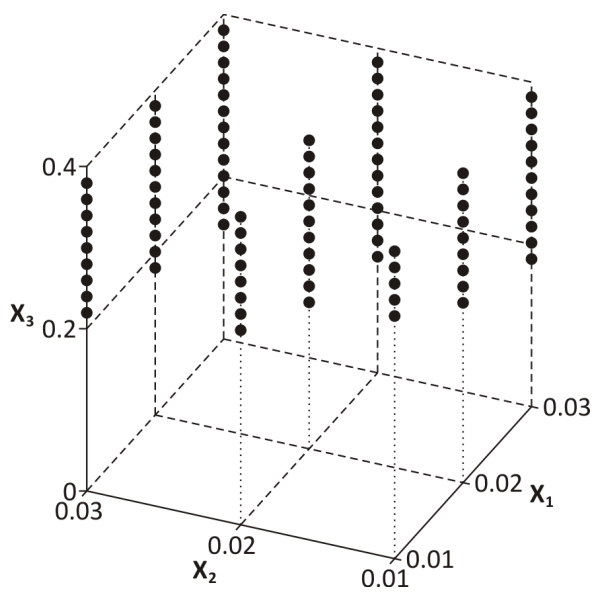

Fig. 3. Map of starting points

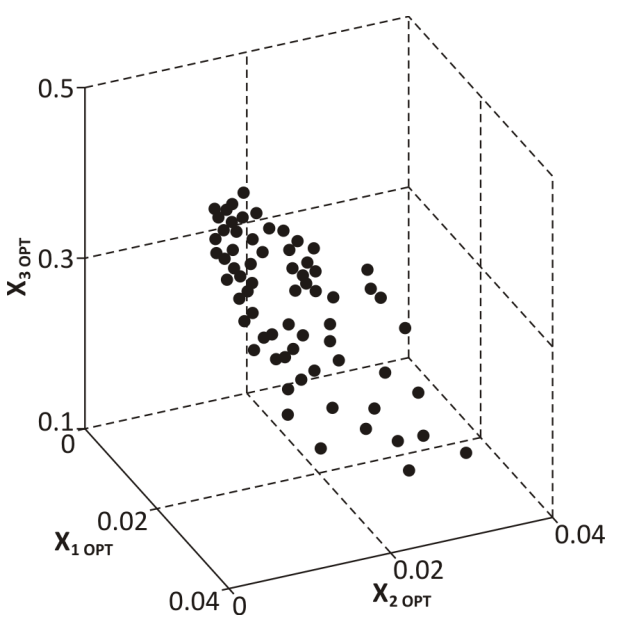

Fig. 4. Map of local optimums

Figure 5 shows a view of the mapped optimum weight of the plate for the different starting points. Based on the analyzed optimization process, the most appropriate starting point was $\mathbf{x}=\left[\begin{array}{lll}0.01 & 0.01 & 0.36\end{array}\right][\mathrm{m}]$ and the subsequently obtained results were globally optimal for the solved area

$$
\mathbf{x}_{O P T}=\left[\begin{array}{lll}
0.014 & 0.010 & 0.364
\end{array}\right][\mathrm{m}]
$$

with parameters - weight $m_{O P T}=1298.5 \mathrm{~kg}$, maximum stress $\sigma_{O P T}=64.6 \mathrm{MPa}$ and maximum deflection $U_{\text {max } \_O P T}=0.00049 \mathrm{~m}$.

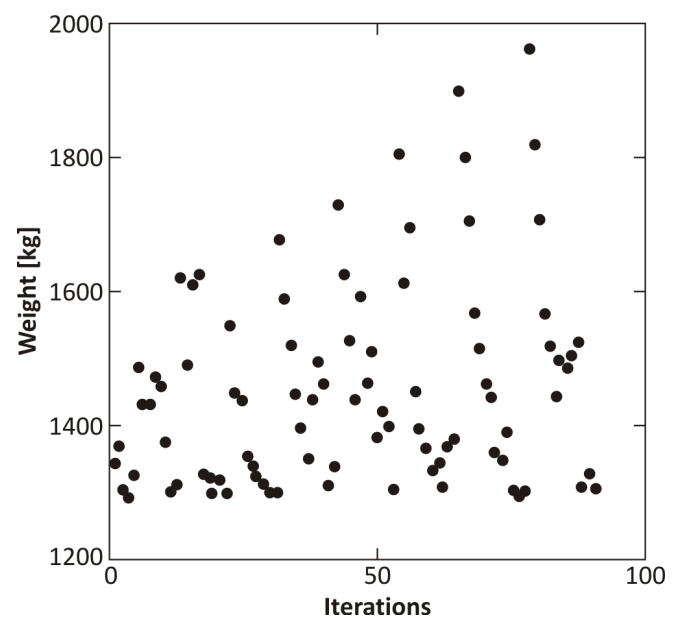

Fig. 5. Map of the optimization results for different starting points 
An overview of the optimization process history for the selected starting point is presented in Figs 6 and 7.
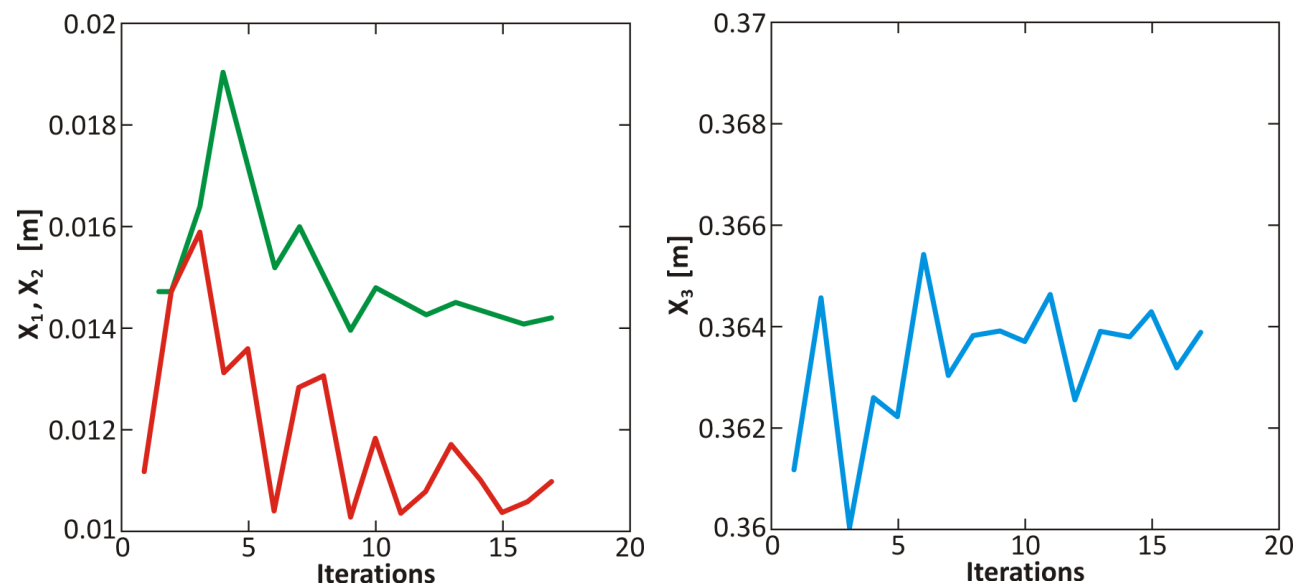

Fig. 6. The convergence process of the optimization variables $X_{1}, X_{2}$ and $X_{3}$
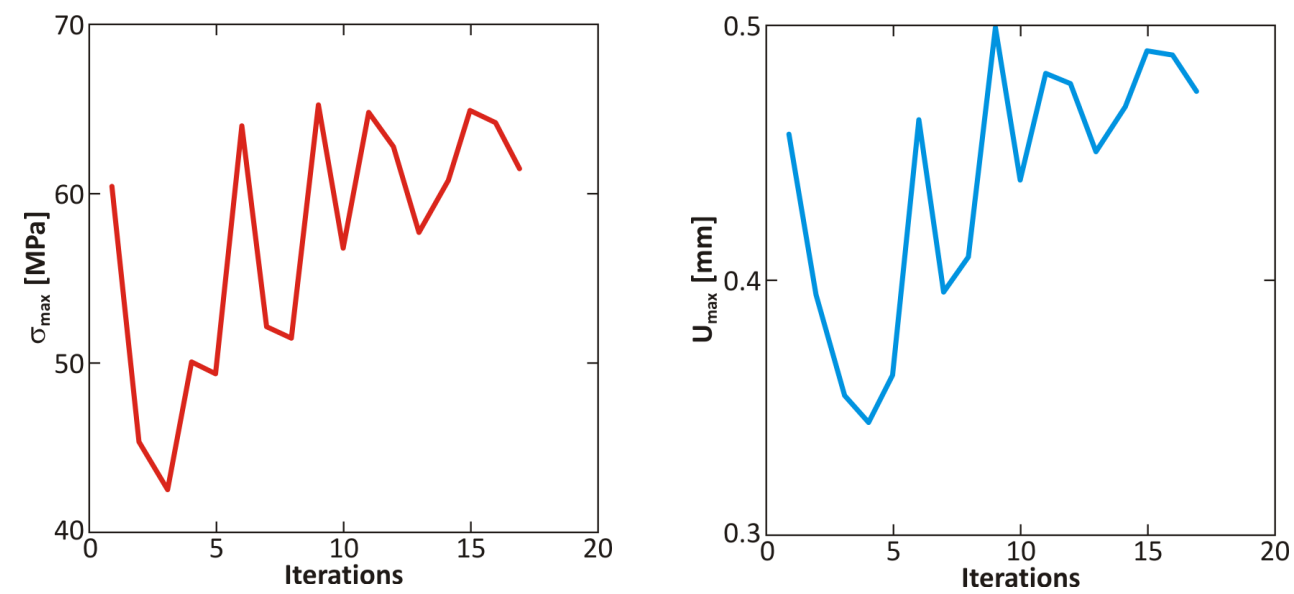

Fig. 7. The convergence process of maximum stress and deflection

The equivalent stress according to the von Mises theory for the selected starting point is presented in Fig. 8. 

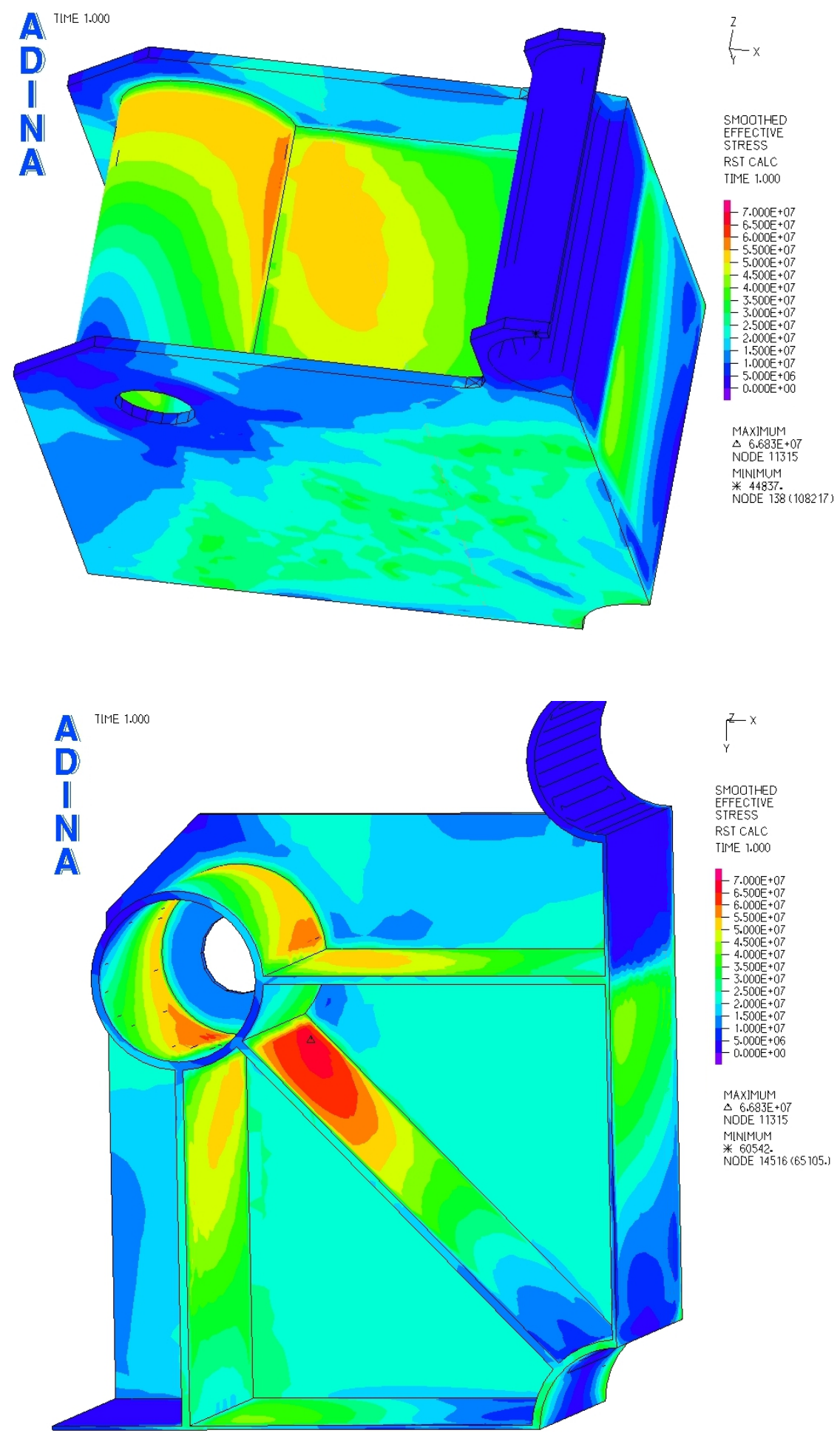

Fig. 8. The equivalent stress according to von Mises theory $[\mathrm{Pa}]$ 


\section{Conclusions}

The process of optimizing the press sandwich plate as an alternative design solution resulting in a reduction of its weight is presented in the article. In the course of solving the problem it has been shown that the starting point significantly affects the final result. This has prompted the authors to mapping the influence of starting values of optimization parameters to the weight and limiting conditions.

The global optimum from the set of local optimums calculated in the investigated area of interest was obtained on the basis of a series of controlled analyzes. The multisoftware approach using ADINA and MATLAB software and the parametric model were used to perform FE analyzes. In addition to the presented calculation methodology for the optimization of extremely stressed sandwich plates, the aim of the paper was to draw attention to the inexplicability in choosing the starting values of the optimization parameters.

This work was supported by KEGA 037ŽU-4/2018 "Implementation of effective computational tools into modern information and communication technologies with the aim of increasing competencies of graduates of technical studies".

\section{References}

1. K. J. Bathe, Finite Element Procedures. (Prentice Hall, New Jersey, 1982)

2. L. Jakubovičová, M. Sága, Computational analysis of contact stress distribution in the case of mutual stewing of roller bearing rings. Novel Trends in Production Devices and Systems, Applied Mechanics and Materials 474, 363-368 (2014)

3. M. Dudziak, A. Kołodziej, G. Domek, K. Talaśka, Multi-angularity - identification of parameters and compatibility conditions of the axisymmetric connection with form deviations. Procedia Engineering 177, 431-438 (2017)

4. M. Sága, M. Vaško, N. Čuboňová, W. Piekarska, Optimisation Algorithms in Mechanical Engineering Applications. (Pearson Education Limited, 291 p., 2016)

5. A. Sapietová, V. Dekýš, M. Sapieta, P. Pecháč, Application of computational and design approaches to improve carrier stability. Procedia Engineering 96, 410-418 (2014)

6. I. Kuric, V. Bulej, M. Sága, P. Pokorný, Development of simulation software for mobile robot path planning within multilayer map system based on metric and topological maps. International Journal of Advanced Robotic Systems 14 (6) (2017)

7. MATLAB. [online] https://www.mathworks.com/products/matlab.html

8. I. Pobočíková, Z. Sedliačková, M. Michalková, Application of four probability distributions for wind speed modeling. Procedia Engineering 192, 713-718 (2017)

9. W. Spendley, G. R. Hext, F. R. Himsworth, Sequential Application of Simplex Designs in Optimization and Evolutionary Operations. Technometrics 4, 441-461 (1962)

10. J. A. Nelder, R. Mead, A Simplex Method for Function Minimization. Computer J. 7, 308-313 (1965)

11. ADINA, Theory and Modelling Guide. [help manual] (Watertown, 2010)

12. L. Žul'ová, R. Grega, J. Krajňák, G. Fedorko, V. Molnár, Optimization of noisiness of mechanical system by using a pneumatic tuner during a failure of piston machine. Engineering Failure Analysis 79, 845-851 (2017) 
13. J. Flizikowski, M. Macko, J. Czerniak, A. Mrozinski, Implementation of genetic algorithms into development of mechatronic multi-edge's grinder design. ASME 2011 International Mechanical Engineering Congress and Exposition, Denver, Colorado, USA, November 11-17, 2011, 1227-1235 (2011)

14. P. Pecháč, M. Sága, Memetic algorithm with normalized RBF ANN for approximation of objective function and sedondary RBF ANN for error mapping. Procedia Engineering 177, 540-547 (2017)

15. W. Piekarska, M. Kubiak, Z. Saternus, Numerical simulation of deformations in T-joint welded by the laser beam. Archives of Metallurgy and Materials 58 (4) 1391-1396 (2013)

16. M. Bara, T. Kmita, J. Korzekwa, Microstructure and properties of composite coatings obtained on aluminium alloys. Archives of Metallurgy and Materials 61 (3), 1107-1112 (2016)

17. J. Vavro, J. Vavro Jr., P. Kováčiková, R. Bezdedová, The optimisation of the materials properties for the passenger cars in dependence on defect distribution at the dynamic loading. Procedia Engineering 136, 114-119 (2016)

18. A. Vaško, L. Hurtalová, M. Uhríčik, E. Tillová, Fatigue of nodular cast iron at high frequency loading. Materialwissenschaft und Werkstofftechnik 47 (5-6), 436-443 (2016)

19. R. Halama, A. Markopoulos, F. Fojtik, M. Fusek, Z. Poruba, J. Famfulík, Effect of stress amplitude on uniaxial ratcheting of aluminum alloy 2124-T851. Materialwissenschaft und Werkstofftechnik 48 (8), 814-819 (2017) 